\title{
An Iterative Solution Approach to Coupled Heat and Mass Transfer in a Steadily Fed Evaporating Water Droplet
}

Lyle School of Engineering,

Southern Methodist University,

Dallas, TX 75205;

ASELSAN Inc.

Ankara 06172, Turkey

e-mail: yakkus@aselsan.com.tr

Barbaros Çetin

Mechanical Engineering Department,

I.D. Bilkent University,

Ankara 06800, Turkey

e-mail: barbaros.cetin@bilkent.edu.tr

Zafer Dursunkaya ${ }^{1}$

Department of Mechanical Engineering,

Middle East Technical University,

Ankara 06800, Turkey

e-mail: refaz@metu.edu.tr
Inspired by the thermoregulation of mammals via perspiration, cooling strategies utilizing continuously fed evaporating droplets have long been investigated in the field, yet a comprehensive modeling capturing the detailed physics of the internal liquid flow is absent. In this study, an innovative computational model is reported, which solves the governing equations with temperature-dependent thermophysical properties in an iterative manner to handle mass and heat transfer coupling at the surface of a constant shape evaporating droplet. Using the model, evaporation from a spherical sessile droplet is simulated with and without thermocapillarity. An uncommon, nonmonotonic temperature variation on the droplet surface is captured in the absence of thermocapillarity. Although similar findings were reported in previous experiments, the temperature dip was attributed to a possible Marangoni flow. This study reveals that buoyancy-driven flow is solely responsible for the nonmonotonic temperature distribution at the surface of an evaporating steadily fed spherical water droplet. [DOI: 10.1115/1.4042492]

Keywords: droplet evaporation, steadily fed droplet, coupling heat and mass transfer, thermocapillarity, buoyancy

\section{Introduction}

Droplet evaporation is a process encountered in nature (e.g., human perspiration) and in many technological applications (e.g., inkjet printing, DNA mapping) [1-4]. Due to the high latent heat of vaporization during phase change, large amounts of heat can be removed by evaporation, which makes droplet evaporation a useful tool in cooling applications [5,6]. Reliable and continuous cooling requires the droplets to endure without being entirely consumed during the process. Feeding the droplet through an opening in the substrate enables the droplet to maintain its shape during cooling [5,6]. Although researchers have made numerous attempts to simulate the thermal energy transport in the dropwise evaporation problems, the liquid flow inside the droplets was rarely considered before 2000s [7-9]. Liquid motion inside an evaporating droplet can be triggered by two mechanisms: buoyancy and thermocapillarity [10]. The contributions of the buoyancy on the evaporation rate and flow pattern are reported as restricted in comparison with the thermocapillarity [11]. Theoretical predictions of the studies where the effect of thermocapillarity was investigated, yielded a counterclockwise flow pattern originating from the monotonic increase of the surface temperature from the center to the edge $[10,12]$. This monotonic surface temperature gradient was shown to reverse its direction at some critical contact angles [13-15]. Zhang et al. [16], on the other hand, reported that a nonmonotonic surface temperature gradient might exist depending on the configuration of the droplet. In recent studies, the nonmonotonic variation of the surface temperatures and the corresponding flow pattern predictions were further investigated [11,17].

The precise modeling of the energy transfer at the droplet interface is a key factor in building a realistic computational model. There are three energy transfer mechanisms taking place at the surface of an evaporating droplet: (i) evaporative heat (mass) transfer, (ii) convective heat transfer, and (iii) radiative heat

\footnotetext{
${ }^{1}$ Corresponding author

Contributed by the Heat Transfer Division of ASME for publication in the JourNAL of Heat Transfer. Manuscript received June 21, 2018; final manuscript received December 21, 2018; published online January 30, 2019. Assoc. Editor: Milind A. Jog.
}

transfer. Although the first one is responsible for the majority of the energy transfer, the other two can become effective especially when the temperature difference between the interface and ambient increases. In the literature, different approaches were taken to model the evaporative mass flux. As being the most popular, $\mathrm{Hu}$ and Larson [18] proposed a semi-empirical correlation as a function of contact angle based on the well-known studies of Deegan et al. $[19,20]$. During the derivation of this correlation, $\mathrm{Hu}$ and Larson assumed the phase equilibrium in a quasi-steady process and solved solely the diffusion equation for the gas phase to calculate the interfacial mass flux from the concentration gradient of vapor based on the assumption of isothermal liquid-vapor interface. In addition, other correlations were proposed using the analogies between heat and mass transfer in different studies $[10,12,21-23]$. Moreover, kinetic theory was utilized in the estimation of the evaporative mass flux [24]. Conservation equations in gas domain, including the mass diffusion, were solved to calculate the local evaporation flux through concentration gradient at the droplet surface $[11,25]$.

Convective heat transfer also contributes to the energy transport at the droplet interface. In the absence of a forced flow of surrounding gas, natural convection of the gas phase drives the convective heat transfer from the interface. In models resolving sessile drop evaporation, the importance of buoyancy was experimentally confirmed by Carle et al. [26]. Although some studies tried to probe the effect of buoyancy of the surrounding gas $[10,11,22,27]$, many studies neglected its presence. Radiative heat transfer, on the other hand, has been neglected in almost every study in the literature of evaporating droplet modeling.

The computational model developed in this study considers all energy transfer mechanisms at the droplet surface in a comprehensive manner: (i) evaporative mass flux is calculated following the mass transfer correlation and heat-mass coupling analogy proposed in Ref. [10], (ii) convective heat transfer through the interface is estimated based on a relevant natural convection correlation [28], and (iii) energy radiating from the interface to its cooler surroundings is calculated based on Stefan-Boltzmann law. The boundary conditions at the droplet interface are functions of 
the interfacial temperature distribution, which is unknown a priori. Therefore, the present work develops a solution methodology in which boundary conditions are updated using the computed interfacial temperature distribution. During the simultaneous solution of the governing equations, a finite element method (FEM)-based solver of COMSOL MULTIPHYSICS software is utilized and an iterative scheme is implemented using the interface, Livelink ${ }^{\mathrm{TM}}$, for MATLAB. This approach enables coupled heat and mass transfer simulations without writing a new code base, by using the number crunching and meshing capability of a state-of-the-art simulation software to solve a free boundary problem. Moreover, the computational model utilizes temperature-dependent thermophysical properties, including surface tension, in the solution of the governing equations to simulate the thermocapillarity and buoyancy effects, precisely. It should be noted that this approach eliminates the need for the application of the Boussinesq approximation, which was a common assumption in previous studies [10,11,27].

A preliminary application of the proposed model was the modeling of evaporation from a spherical (with 90 deg contact angle), sessile, and continuously fed (by liquid injection) droplet in the absence of thermocapillary effect [29], where a temperature well was found on the droplet surface for high substrate heating rates. To address this observation, the preliminary model has been improved by adding the effects of thermocapillary flow and radiative heat transfer to the surroundings in this study. The model is, then, applied to a steadily fed spherical water droplet placed on a heated substrate [30], to enable a direct comparison of predicted and experimental interface temperature variation.

\section{Modeling}

Continuous evaporation from a sessile water droplet located on a heated substrate is modeled. The droplet is assumed to have a spherical shape, which enables the implementation of a twodimensional axisymmetric model in spherical coordinates, as shown in Fig. 1. The spherical geometry of the droplet is preserved by continuous liquid feeding from a concentric inner half sphere. A constant substrate temperature is assigned, and the feeding water is assumed to be in thermal equilibrium with the heated substrate.

2.1 Governing Equations and Boundary Conditions. Steady forms of the conservation of mass, momentum, and energy are solved numerically by means of the FEM solver of COMSOL MULTIPHYSICS software for the liquid domain shown in Fig. 1

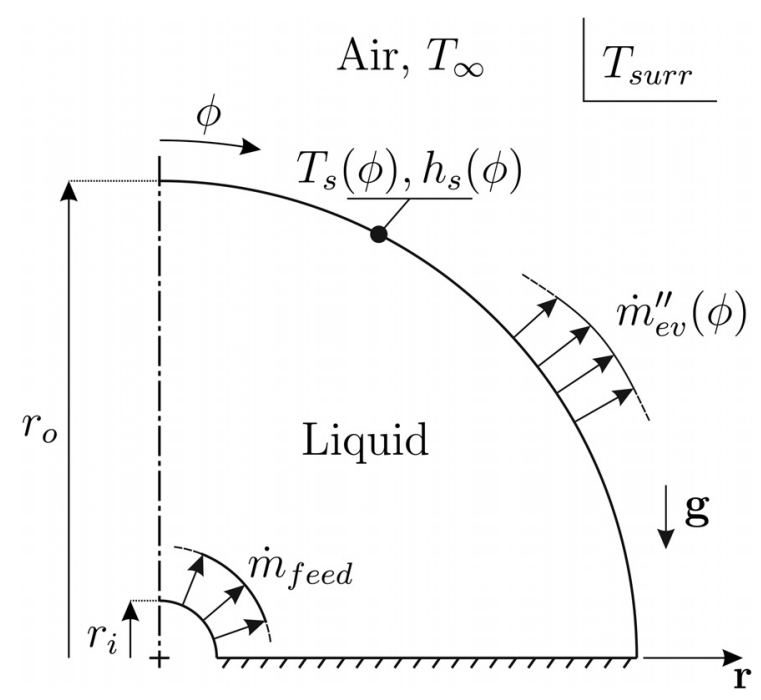

Fig. 1 Problem domain

$$
\begin{gathered}
\nabla \cdot(\rho \mathbf{u})=0 \\
\rho(\mathbf{u} \cdot \nabla) \mathbf{u}=-\nabla p+\nabla \cdot \overline{\bar{\tau}}+\rho \mathbf{g} \\
\rho c_{p} \mathbf{u} \cdot \nabla T=\nabla \cdot(k \nabla T)+\overline{\bar{\tau}}: \nabla \mathbf{u}
\end{gathered}
$$

where $\overline{\bar{\tau}}$ is the deviatoric stress tensor defined as $\mu\left(\partial u_{i} / \partial x_{j}\right.$ $\left.+\partial u_{j} / \partial x_{i}\right)$; and $\mu, c_{p}$, and $k$ are dynamic viscosity, specific heat, and thermal conductivity of the liquid water and all liquid properties, including the density, are defined as thermally dependent in the solver. The two-dimensional axisymmetric model ensures the symmetry boundary condition along the center line of the droplet. On the substrate, no-slip and constant temperature boundary conditions are defined. The inlet boundary of the domain, where a continuous supply of water is fed, is at the same temperature as the substrate, $T_{w}$. The velocity of the water at the inlet, $\overline{\mathbf{u}}_{\text {in }}$, is assumed to be uniform. The distributions of liquid velocity, $\mathbf{u}_{\mathbf{s}}$, convection coefficient, $h_{s}$, and evaporative mass flux, $\dot{m}_{\mathrm{ev}}^{\prime \prime}$, at the liquid-gas interface are unknown a priori; therefore, these quantities at the interface are evaluated in an iterative algorithm whose details are given in Sec. 3. The boundary conditions associated with Eqs. $(1 a)-(1 c)$ can be summarized as

$$
\begin{gathered}
\partial_{\phi} \mathbf{u}=0, \partial_{\phi} T=0 \text { at } \phi=0 \\
\mathbf{u}=0, T=T_{w} \text { at } \phi=\pi / 2 \\
\mathbf{u}=\overline{\mathbf{u}}_{\mathrm{in}}, T=T_{w} \text { at } r=r_{i} \\
\mathbf{u}=\mathbf{u}_{\mathbf{s}},-k \partial_{r} T=\dot{m}_{\mathrm{ev}}^{\prime \prime} h_{\mathrm{fg}}+h_{s}\left(T-T_{\infty}\right) \\
+\sigma \varepsilon\left(T^{4}-T_{\text {surr }}^{4}\right) \text { at } r=r_{o}
\end{gathered}
$$

where $\sigma$ is the Stefan-Boltzmann constant and $\epsilon$ is emissivity of water, which is 0.97 for the interface of pure water [31]. The temperature of the surroundings, $T_{\text {surr }}$ is assumed to be in thermal equilibrium with the ambient air, i.e., $T_{\text {surr }}=T_{\infty}$. Calculations of the evaporative mass flux, convection coefficient, and velocity distribution at the liquid-gas interface, on the other hand, require a close examination of energy and mass balances at the interface, whose details are provided in Secs. 2.2 and 2.3.

2.2 Energy Balance at the Interface. The boundary condition at the interface links the fluid flow and heat transfer of the liquid to thermal and mass transport occurring in the surrounding gas. The higher temperature of the liquid in conjunction with the phase change at the interface creates a buoyancy, inducing a natural convection flow field on the surrounding gas. The characterization of this field, extending far away from the liquid surface, is both complex and computationally demanding even prohibitively expensive. Therefore, in this study - as in many such problems - the conditions at the interface are described using correlations pertinent to this problem.

Calculation of the evaporation rate is crucial in the modeling of droplet evaporation and several correlations are analyzed to determine how to accurately model the evaporative mass flux. In the case of pinned droplets, Deegan et al. $[19,20]$ suggested a simple curve fit for the evaporation rate along the interface, $J(r)=J_{0}\left(1-r^{2} / R^{2}\right)^{-\lambda}$, where $J_{0}$ was related to vapor mass diffusion and $\lambda$ was a fitting parameter accounting for the nonuniformity of the evaporation rate along the droplet interface. Hu and Larson [18] provided semi-empirical correlations of $\lambda(\theta)$ and $J_{0}(\theta)$ as functions of contact angle. In this study, Hu and Larson solved only the diffusion equation for the gas phase and calculated the interfacial mass flux from the concentration gradient of vapor based on the assumption of an isothermal liquid-vapor interface. Therefore, their correlations did not include the possible effects of natural convection of the vapor and temperature variations of the interface temperature resulting from evaporative cooling.

Droplet evaporation is influenced by the dynamics of the vapor phase, particularly when the substrate is substantially warmer than 
the surroundings [26]. An evaporative mass flux correlation based on a constant interface temperature assumption is not applicable for the current study due to the significant temperature variation along the interface. Modeling of the most realistic energy transfer at the interface requires the simultaneous solution of heat and diffusion equations at the vapor phase [11,25]. However, application of an empirical heat and mass transfer correlation simplifies the problem by eliminating the need to solve for the heat equation in the vapor phase [12,21-23]. On the other hand, Laplace solution of the vapor field was used $[13,18,32]$ for the calculation of mass transfer at the interface. However, this study does not specifically aim to model the gas flow in the surroundings, but the effect of gas motion must be included to the evaporation model to ensure accuracy. Therefore, the mass transfer correlation and heat-mass coupling analogy is used, which was also utilized in [10]

$$
\dot{m}_{\mathrm{ev}}^{\prime \prime}=h_{m}\left[\rho_{\mathrm{vap}, \mathrm{sat}}\left(T_{s}\right)-\rho_{\mathrm{vap}, \infty}\right]
$$

where $h_{m}$ is the mass transfer coefficient and it is defined as a function of local interfacial temperature, $T_{s}(\phi)$. The mass transfer coefficient is obtained using the analogy between heat and mass transfer [33]

$$
h_{m}(\phi)=\frac{h_{s}(\phi)}{\rho_{\text {air }} c_{p, \text { air }} \mathrm{Le}^{2 / 3}}
$$

where $\rho_{\text {air }}$ and $c_{p \text {,air }}$ are density and specific heat of the surrounding air, respectively, and Le is the Lewis number defined as, $\mathrm{Le}=\alpha_{\text {air }} / D$, where $\alpha_{\text {air }}$ and $D$ are the thermal and mass diffusivities of vapor within the air, respectively. $h_{s}(\phi)$ is the convection heat transfer coefficient as a function of local interfacial temperature $T_{s}(\phi)$. In the absence of a forced flow of surrounding gas, natural convection of the gas phase drives the convective heat transfer from the interface. Therefore, in order to obtain a reliable estimate for the convective heat transfer coefficient, the empirical correlation derived for an immersed sphere in a surrounding fluid [28] as proposed by Ref. [10] is used

$$
h_{s}(\phi)=\frac{k_{\mathrm{air}}}{2 r_{0}}\left[2+\frac{0.589 \mathrm{Ra}^{1 / 4}}{\left[1+(0.469 / \mathrm{Pr})^{9 / 16}\right]^{4 / 9}}\right]
$$

where $k_{\text {air }}$ is the thermal conductivity of the surrounding air; Ra is the Rayleigh number defined as $\mathrm{Ra}=g \beta\left(2 r_{0} / 3\right)^{3}\left(T_{s}-T_{\infty}\right)$ $/\left(\nu_{\text {air }} \alpha_{\text {air }}\right)$; Pr is the Prandtl number; $\beta, \nu_{\text {air }}$, and $\alpha_{\text {air }}$ are thermal expansion coefficient, kinematic viscosity, and thermal diffusivity of air, respectively. The application of this correlation to model the convective heat loss from the droplet interface was attempted in Ref. [10] with the assumption of a small temperature difference between the droplet interface and surrounding air, pushing the correlation to its conduction limit. This study considers the complete correlation, which takes into account the temperature variation along the interface. It should be noted that the correlation given in Eq. (5) is valid for a sphere hanging in a fluid environment such that ambient fluid is flowing upward keeping contact with the interface of the sphere starting from the bottom, for a constant surface temperature. Therefore, a discrepancy between the experimental and theoretical results can be expected, and in order to match the volumetric flow rate measured in the experiments, a scaling of the theoretical value by a factor within each simulation, is applied.

Energy transport is not exclusively due to the phase change at the droplet interface, since convective and radiative heat transfer mechanisms may also contribute to the energy transport from the droplet interface. The current study includes the convective energy transfer from the droplet interface using the convection coefficient proposed in Eq. (5). Radiative heat transfer from the droplet interface to the cooler surrounding, which is assumed to be in thermal equilibrium with air, is also included in the energy balance at the interface.

2.3 Mass and Force Balance at the Interface. The mass conservation at the droplet interface requires the balance of outflow and evaporating mass flow

$$
\mathbf{u}_{\mathrm{s}} \cdot \mathbf{n}=\frac{\dot{m}_{\mathrm{ev}}^{\prime \prime}(\phi)}{\rho(\phi)}
$$

where $\mathbf{n}$ and $\rho(\phi)$ are unit normal vector and density of the liquid at the interface as a function of the interfacial temperature, $T_{s}(\phi)$, respectively.

A nonuniform distribution of the interfacial temperature can initiate thermocapillary (or Marangoni) convection due to the imbalance of surface tension forces. Since the substrate in the experimental work was heated and there was a temperature gradient tangential to the interface, a tangential force balance is included to account for the surface tension force. Moreover, the shear stress induced by the gas phase can be neglected due to lower viscosity of the gas, which results in the following balance for tangential forces:

$$
\nabla \gamma \cdot \mathbf{t}=-\mathbf{n} \cdot \overline{\bar{\tau}} \cdot \mathbf{t}
$$

where $\gamma$ and $\mathbf{t}$ are surface tension of the liquid and unit tangent vector tensor of the liquid, respectively. Previous studies $[10,13,14,17]$ assumed a linear variation of the surface tension with temperature, but due to the importance of the thermocapillary convection in this study, a second-order polynomial is used, which is fitted to the values obtained by the precise measurements of Ref. [34], and the temperature derivative of the surface tension is calculated based on the local temperatures at the interface.

The normal force balance at the droplet interface is between the interface liquid pressure, interface vapor pressure, and the capillary pressure. Assuming a uniform constant pressure in the gas phase, higher radius of curvature values is expected near the top of the droplet as given by the Young-Laplace equation. Therefore, a flattening is expected near the top region of the droplet, which alters the shape of the droplet. The ratio of gravitational forces to surface tension forces is given by the Bond number

$$
\mathrm{Bo}=\frac{m_{\mathrm{drop}} g}{L_{\mathrm{cl}} \gamma}=\frac{\rho g r_{o}^{2}}{3 \gamma}
$$

where $m_{\mathrm{drop}}$ and $L_{\mathrm{cl}}$ are the mass of a half droplet and length of the of the droplet, respectively. The Bond number for the water droplet studied $\left(r_{i}=0.175 \mathrm{~mm}\right.$ and $\left.r_{o}=2.5 \mathrm{~mm}\right)$ in the current work is approximately 0.28 , which indicates that the effect of gravity is small and the droplet preserves its spherical shape.

2.4 Meshing of the Computational Domain. Meshing is performed by COMSOL, which results in 15,371 triangular and 318 quadrilateral elements. Grid independence is tested using three different sets of grids. The performance of each mesh is tested based on the volume-averaged temperature of the droplet. Results exhibit that simulations yield almost identical results for all mesh configurations tested, within $0.03^{\circ} \mathrm{C}$ difference, which assured the grid-independence of the simulations.

\section{Computational Scheme}

One of the main challenges in the modeling of droplet evaporation is that boundary conditions at the liquid-gas interface are functions of the interface temperature, which is unknown a priori. Consequently, distributions of liquid velocity, the convective heat transfer coefficient, and evaporative mass flux cannot be directly defined at the interface. The proposed model applies an iterative two-loop computational scheme (Fig. 2), which enables the 


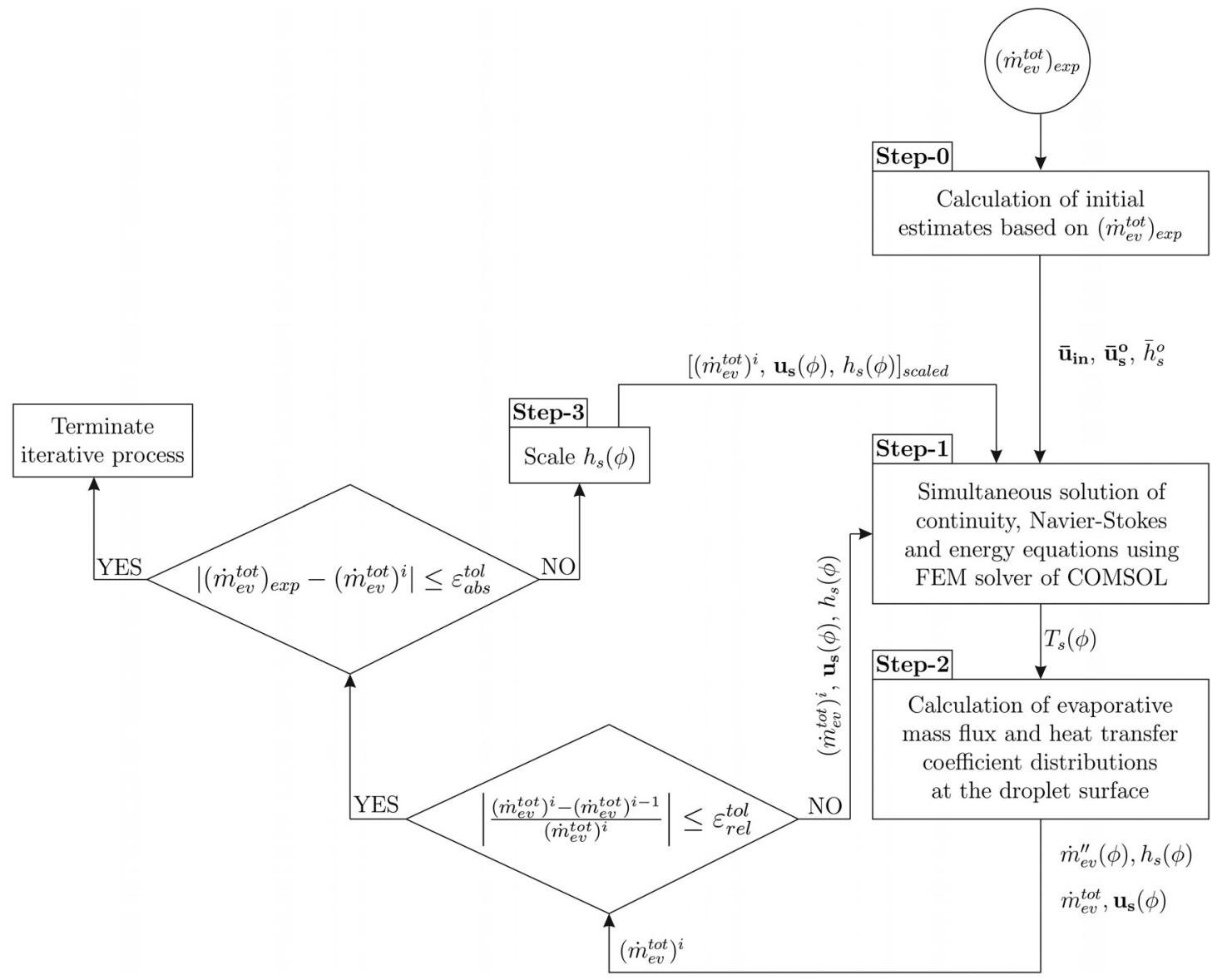

Fig. 2 Flowchart of the computational scheme

simultaneous solution of the steady forms of the mass, momentum, and energy equations with temperature-dependent thermophysical properties via the inner loop and guarantees the total amount of evaporating mass to be equal to the experimentally estimated value via the outer loop. In Secs. 3.1-3.4, each step of the iterative process is explained in detail.

3.1 Step-0: Initialization. In the initialization step, initial estimates of the boundary conditions at the inlet and outlet of the domain-i.e., liquid feeding surface $\left(r=r_{i}\right)$, and liquid-vapor interface $\left(r=r_{o}\right)$ of the droplet-are assigned. A uniform velocity distribution is assumed at the inlet and this average value, which is calculated based on the experimental liquid feeding rate as shown in Eq. (9), is applied for the current model without any modification

$$
\bar{u}_{\mathrm{in}}=\frac{\left(\dot{m}_{\mathrm{ev}}^{\mathrm{tot}}\right)_{\exp }}{A_{\text {in }} \rho\left(T_{w}\right)}
$$

where $A_{\text {in }}$ is the surface area of the inlet surface. It should be noted that the velocity at the inlet is assumed to be perpendicular to the inlet surface. Distribution of the velocity at the liquid-gas interface, on the other hand, is iterated on within the computational scheme and a uniform distribution is only assumed for the initial estimate, which is calculated based on the ratio of the surface areas as follows:

$$
\bar{u}_{s}^{o}=\bar{u}_{\text {in }} \frac{A_{\text {in }}}{A_{\text {out }}}
$$

Based on an initially estimated constant interface temperature, a uniform distribution is assumed for the heat transfer coefficient as the initial guess. This average initial estimate, $\bar{h}_{s}^{o}$, is obtained using Eq. (5) without an application of scaling.

3.2 Step-1: Simultaneous Solution of the Governing Equations. In step-1, continuity, momentum, and energy equations, Eqs. (1a)-(1c), with the corresponding boundary conditions, Eqs. $(2 a)-(2 d)$, are solved simultaneously using the FEM solver of COMSOL software, which enables the thermo-physical properties to be temperature dependent. In this step, solver is applied iteratively to implement the thermocapillarity-induced boundary condition, $\nabla_{t} \gamma=\mu\left(\partial_{t} V_{n}+\partial_{n} V_{t}\right)$, where $V_{n}$ and $V_{t}$ are normal and tangential components of the interface liquid velocity.

3.3 Step-2: Calculations of Evaporative Mass Flux and Heat Transfer Coefficient. In step-2, evaporative mass flux, $\dot{m}_{\mathrm{ev}}^{\prime \prime}(\phi)$, and heat transfer coefficient, $h_{s}(\phi)$, are calculated based on the interface temperature distribution, $T_{s}(\phi)$, which is evaluated in step-1, using Eqs. (3)-(5), where properties of air are at the ambient temperature, $T_{\infty}$. In addition, corresponding normal velocities and the total amount of the evaporating mass are calculated. The updated interface velocity distribution is stored to be used in the next iterative step.

Until convergence, the normal velocities and interfacial temperatures obtained at step- 2 are not equal to the input values to step1. Total evaporated mass from the droplet, obtained at the end of step-2, is used as a criterion of convergence of steps-1 and -2 at the inner loop decision point. Values of normal velocity, $\mathbf{u}_{\mathbf{s}}(\phi)$, and heat transfer coefficient, $h_{s}(\phi)$, are iterated on until the convergence is reached in steps- 1 and -2 .

3.4 Step-3: Scaling of the Heat Transfer Coefficient. The convergence of steps-1 and -2 (the inner loop) does not guarantee 
the convergence of the total evaporated mass to the experimentally observed value. The outer loop decision point checks this convergence and the computational scheme uses the total evaporated mass for the convergence check. If the absolute error is larger than the desired, the amount of evaporating mass should be rescaled appropriately. Therefore, in the current modeling, the heat transfer coefficient is scaled, which updates the total amount of evaporating mass and interface velocities. The computational scheme is concluded when the outer iteration loop converges.

\section{Results and Discussion}

Three different cases are simulated with and without thermocapillarity. While the ambient temperature is $30^{\circ} \mathrm{C}$ for all three cases, substrate temperatures are $39^{\circ} \mathrm{C}$ for case $1,58^{\circ} \mathrm{C}$ for case 2 and $74^{\circ} \mathrm{C}$ for case 3 to enable a comparison with available experiments [30].

Surface temperature predictions with and without the presence of thermocapillarity along with experimental results are given in Fig. 3. Thermocapillarity results in an approximately uniform temperature distribution on the droplet surface, and the stronger convection carries the high temperature fluid toward the apex leading to temperatures higher than those observed in the experiments. The observed temperatures displayed a characteristic minimum between $60 \mathrm{deg}$ and $70 \mathrm{deg}$ polar angles for cases 2 and 3, which is absent in the simulation results with the thermocapillarity. The simulations without the inclusion of the thermocapillarity are also given in Fig. 3, which capture the characteristic temperature wells clearly observed in the experiments. In addition, the monotonic temperature change prediction of case 1 without thermocapillarity is in good agreement with the experiments.

The temperature distribution within the droplet without thermocapillarity is given in Fig. 4(a) with the superimposed streamlines and velocity vectors. Case 1 has the lowest temperature difference between the heated substrate and the ambient. Hot liquid entering the domain moves to the apex of the droplet. In close proximity of the interface, the liquid cools due to heat transfer and evaporation on the surface, and temperatures continue to drop as the colder fluid moves along the interface toward the heated surface under the influence of buoyancy. Near the substrate, fluid is heated due to conduction from the substrate and flows to the feeder section of the droplet where new hot liquid is introduced into the domain. The two hot liquid streams merge and move to the apex of the droplet resulting in a clockwise (CW) rotation. Case 2 has a higher temperature difference between the substrate and ambient compared to that of case 1 . Although both cases have a similar flow pattern, the temperature distribution within the droplet is quite different, as seen in Fig. 4(b). A close examination of Figs. 4(a)-4(c) reveals that for the small temperature difference case (case 1), a temperature stratification exists in the entire domain along the vertical direction. Unlike cases 2 and 3, temperatures in any vertical plane monotonically decrease, resulting in the absence of a temperature well on the interface. The two cases with higher temperature differences (cases 2 and 3), on the other hand, have a more complex temperature distribution due to more intense buoyancydriven convection, and the circulation results in a temperature minimum in the vertical planes beyond the incoming jet, circa $0.6-1.0 \mathrm{~mm}$ away from the line of symmetry in both cases, resulting in a temperature well on the surface. The current simulation yields an intermediate cooler zone above the substrate and near the interface. Case 3 has the highest temperature difference between the heated substrate and ambient. The pattern is similar to the other cases, and the temperature distribution is similar to that of case 2 but the location of the temperature well is shifted toward the substrate side.

The magnitude of the Marangoni numbers in the current study ranges between 2500 and 20,500 well above the critical value of 80 [35], which, in fact, indicates the presence of strong thermocapillary convection. The temperature distribution within the droplet including the effect of thermocapillarity is given in Fig. 5. In all three cases, the liquid circulation is counter clockwise $(\mathrm{CCW})$ in the direction of monotonically decreasing temperatures on the droplet surface. Due to the strong CCW circulation, the incoming fluid is forced to move parallel to the substrate and eventually turns toward the apex at the close proximity of the interface. For cases 2 and 3, due to the higher temperature difference, the incoming fresh fluid separates from the substrate before reaching the interface, which leads to a small region of $\mathrm{CW}$ circulation between the main flow path and the substrate. These are all in accordance with numerous studies in the literature that report the presence of a main $\mathrm{CCW}$ vortex within water droplets with contact angles close to $90 \mathrm{deg}[10,11,16]$. Therefore, the present model predicts that strong Marangoni flow, shown in Fig. 6(a), dominates the buoyancy-induced weak flow, shown in Fig. 6(b), for steadily fed spherical water droplets similar to their drying counterparts with high contact angles. However, the experimentally measured surface temperature distributions of Ref. [30] conform with the calculated temperature variations of the current study only in the absence of thermocapillarity, which led to a nonmonotonic temperature distribution at the interface as evident in Fig. 3. Moreover, current simulations with thermocapillarity demonstrate that a nonmonotonic variation, i.e., a temperature dip, is not possible in the presence of Marangoni flow for a droplet with 90 deg contact angle in conjunction with numerous previous studies $[10-12,15,16]$. It should be noted that the existence of thermocapillary flow for pure water has been contentious in the literature $[36,37]$. Although the temperature derivative of the surface tension of pure water is capable of generating thermocapillary convection, high attraction of water molecules to the surfactants was speculated to hinder the thermocapillary flow [38].

The simulations were also carried out for the constant heat flux boundary condition at the heated substrate. To facilitate a comparison, the heat input was kept identical to the corresponding constant temperature boundary condition case in order for both cases to have the same rate of heat and mass transfer. The temperature and flow fields were almost identical, resulting in similar interpretations for the individual effects.

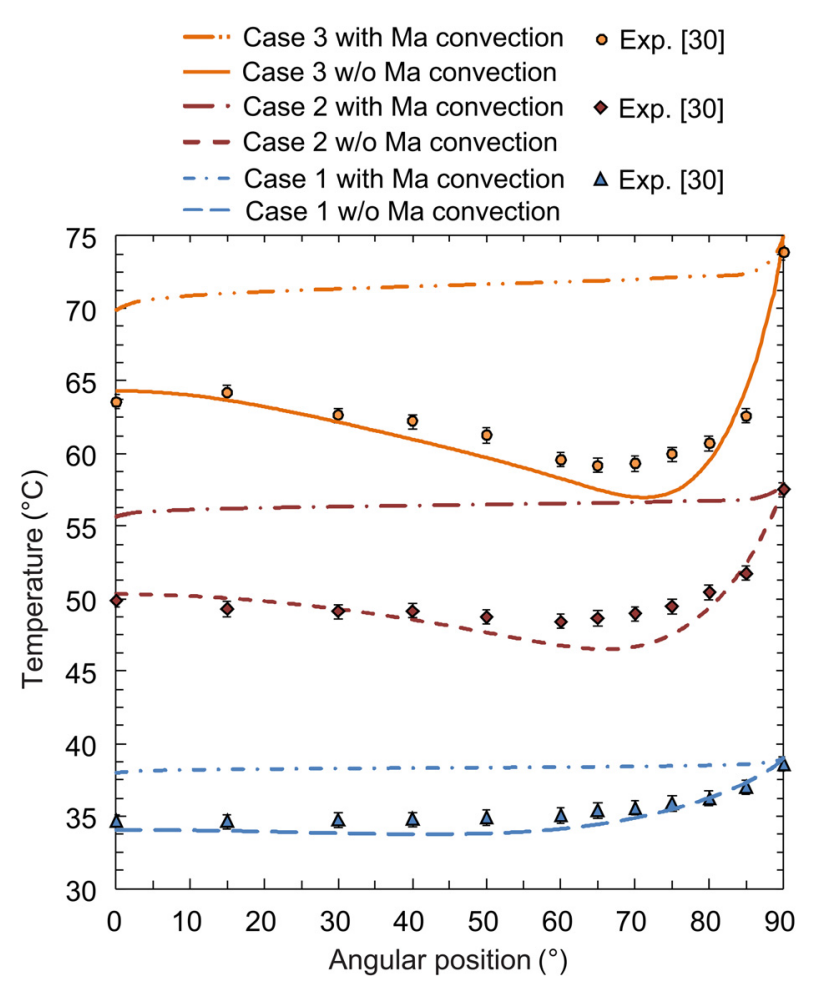

Fig. 3 Measured surface temperatures [30] and computed counterparts simulated with and without thermocapillarity 
4.1 Sensitivity Analysis. A sensitivity analysis is carried out to see the effect of the droplet radius and the volumetric flow rate, two main parameters that affect the temperature field, which are prone to measurement uncertainties. To estimate the effect of geometry change and the flow rate on the surface temperature distribution, additional simulations are performed based on different droplet radii and flow rates, the results of which are given in Fig. 7 for case 3 . The uncertainty of temperature predictions based on $\pm 4 \%$ radius change is estimated to be within $\pm 1{ }^{\circ} \mathrm{C}$. Therefore, local radius of curvature changes on the droplet surface might be a possible reason for some of the local deviations between the experimental data and simulation results. A similar analysis of uncertainty based on 7\% flow rate change also results in an approximately $\pm 1{ }^{\circ} \mathrm{C}$ change in surface temperature estimates, bringing the simulated results closer to experimental observations for the higher flow rate value. The predictions capture the trends effectively, particularly the temperature wells observed in experiments.

4.2 Energy Transport and Assessment of Experimental Measurements at the Droplet Surface. The presence of convection cells within the droplet suggests that conduction is not the sole mechanism carrying energy to the droplet surface. Substantial experimental effort has been devoted to quantify the contribution of internal convection to the energy transport at the liquid/vapor interface of water [21,30,36,39-41]. While the radial temperature measurements were used to determine the conduction heat transfer to the interface, the concept of surface thermal capacity was proposed and applied to establish the energy balance at an interface with tangential velocity due to thermocapillarity $[39,40]$. Computational modeling, on the other hand, enables making accurate predictions especially in the close proximity of the interface, where the spatial resolution of experimental temperature measurements is restricted by the physical dimension of measurement probe. In order to predict the contribution of internal circulation to the heat transfer at the interface with/without thermocapillarity and to assess the experimental temperature measurements beneath the interface, the energy balance in a finite control volume contiguous to the droplet interface (Fig. 8) is made. Energy is transferred to the control volume by diffusion (conduction) and advection mechanisms, which are denoted by $\dot{q}^{\text {cond }}$ and $\dot{q}^{\text {adv }}$ in Fig. 8, respectively. The net amount of energy carried to the control volume, which is equal to the rate of conduction at the interface $\left(\dot{q}_{r_{0}}^{\text {cond }}\right)$, does not only provide the energy required for the phase change $\left(\dot{q}^{\text {ev }}\right)$, but is also dissipated to surroundings by diffusion $\left(\dot{q}^{\text {diff }}\right)$ and radiation $\left(\dot{q}^{\mathrm{rad}}\right)$. It should be noted that diffusive heat transfer from the interface is simply the conduction on the vapor side, which is usually accounted for by the definition of the "convective heat transfer coefficient" due to the complexity of solving the energy equation in the gas phase.
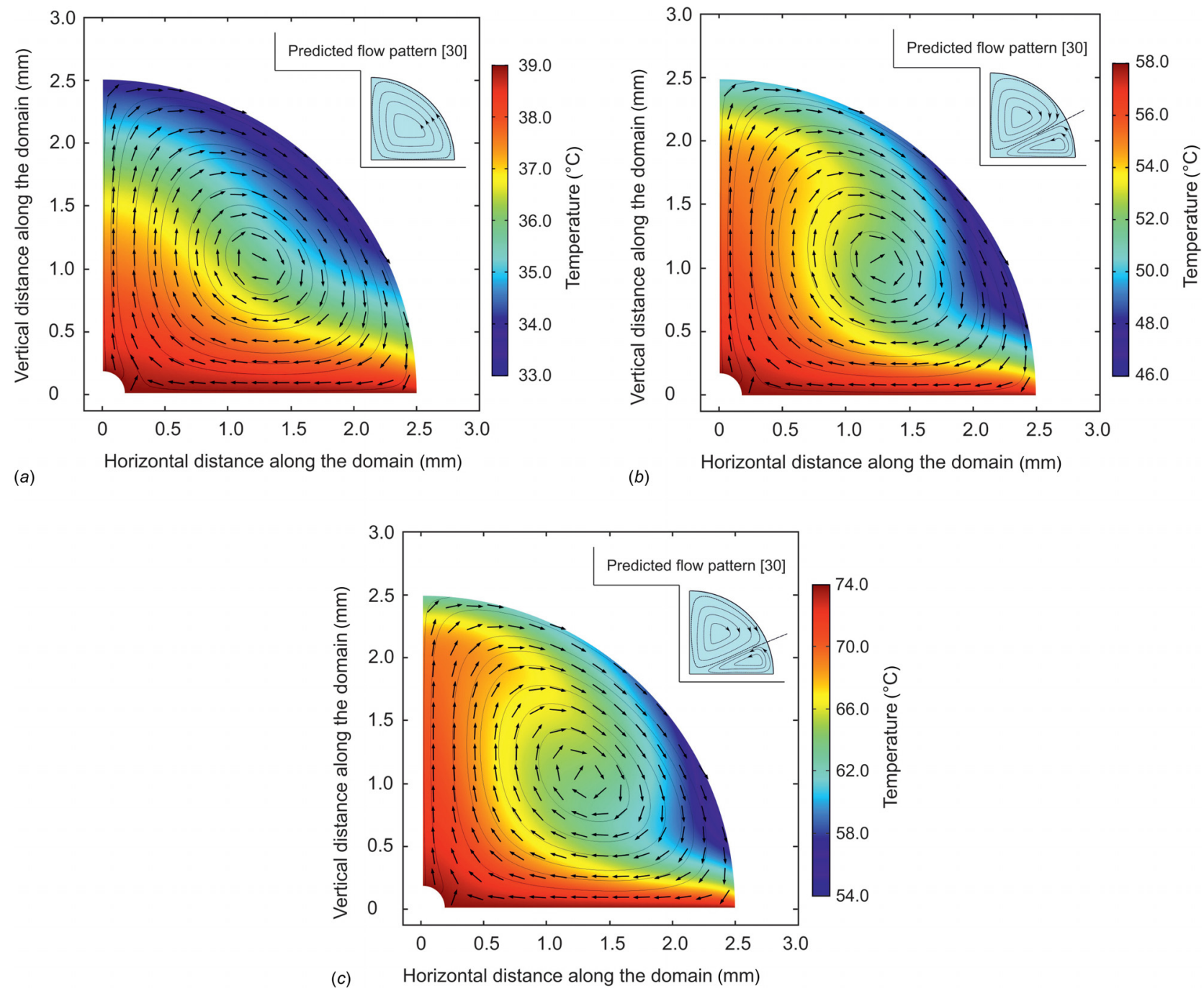

Fig. 4 Temperature distribution, streamlines, and velocity vectors without thermocapillarity: (a) case 1, (b) case 2, and (c) case 3 

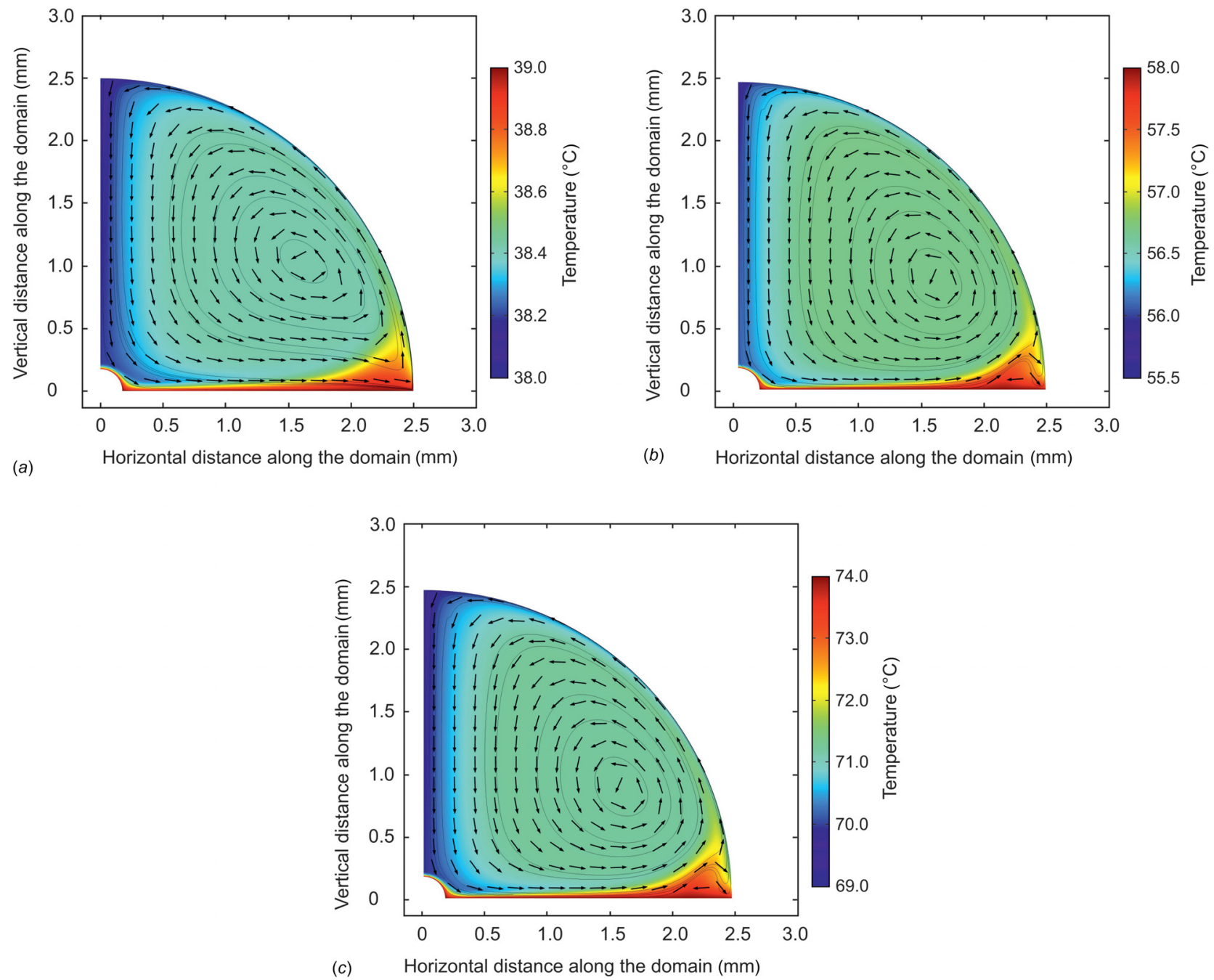

Fig. 5 Temperature distribution, streamlines, and velocity vectors with thermocapillarity: (a) case 1, (b) case 2, and (c) case 3

Energy transport at the interface is investigated for case 3 , where the circulation is strongest due to higher superheat, with and without the presence of thermocapillarity. The depth of the control volume is selected as $\Delta r=50 \mu \mathrm{m}$, which was the nearest measurement distance to the interface in Ref. [30]. Regardless of the effective mechanism inducing the internal flow, the tangential velocity dominates the radial velocity at this depth as shown in Figs. $9(a)$ and $9(b)$. Therefore, the contribution of radial advection

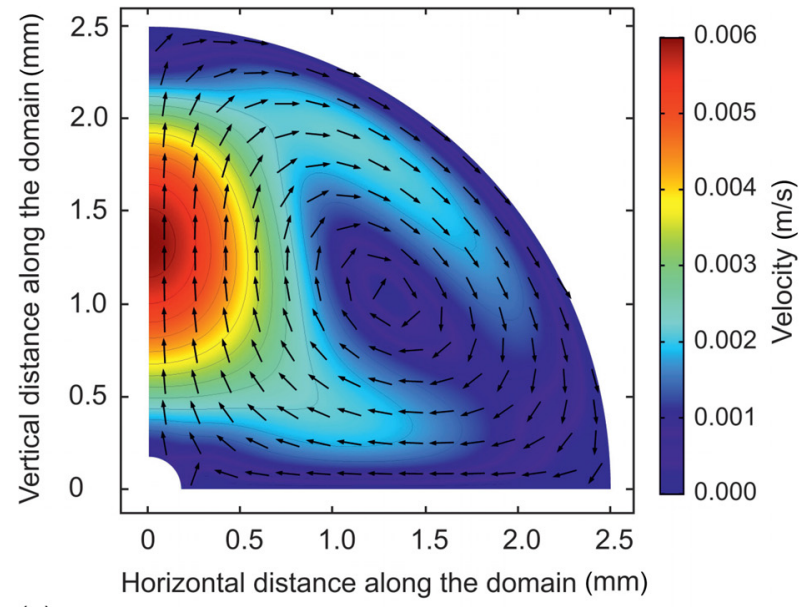

(a)

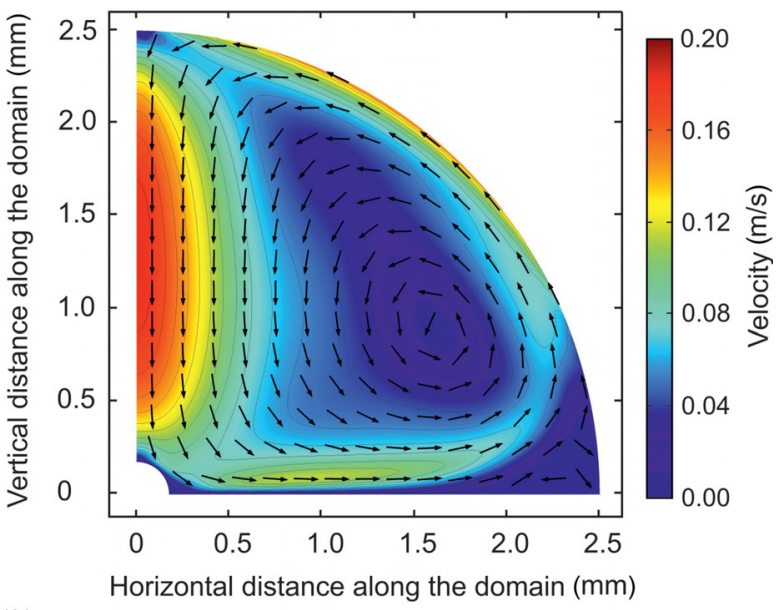

(b)

Fig. 6 Velocity distribution and velocity vectors in the droplet with and without Marangoni convection: (a) case 3 w/o Ma convection and $(b)$ case 3 with Ma convection 


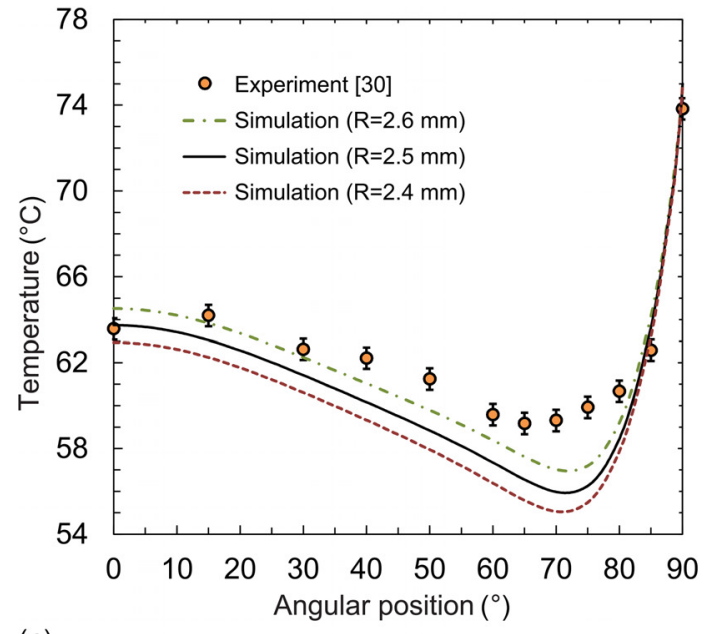

(a)

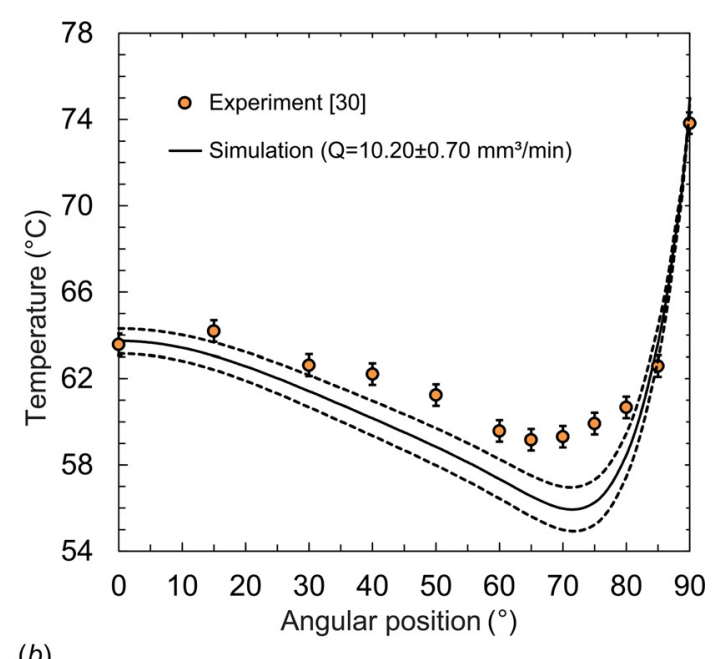

(b)

Fig. 7 Distribution of interface temperature for different droplet radii and flow rates: (a) case 3 and (b) case 3

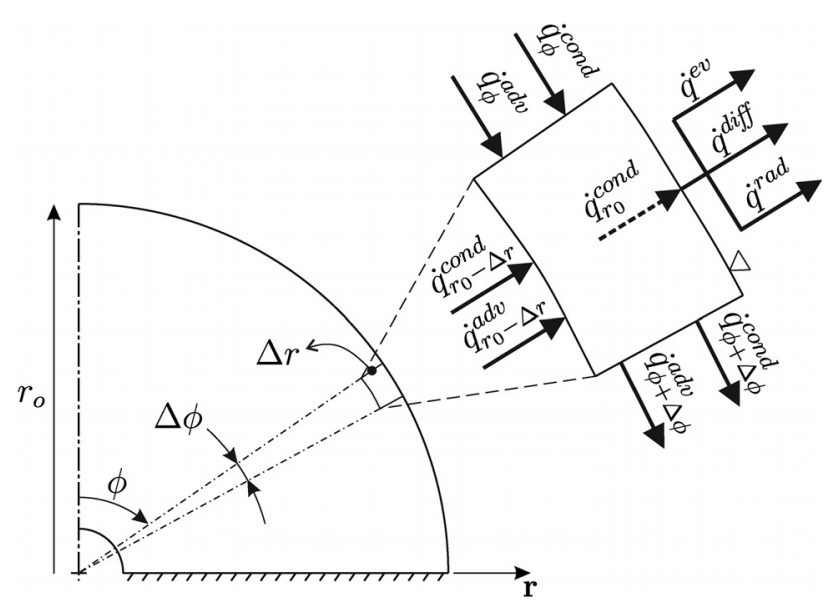

Fig. 8 Energy balance on a sample control volume beneath the interface

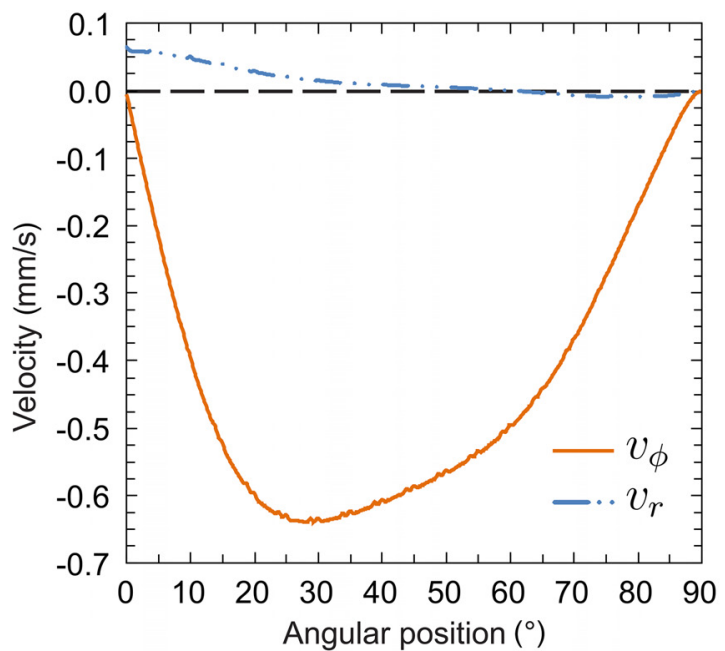

$\left(\dot{q}_{r_{0}-\Delta r}^{\text {adv }}\right)$ is small compared to its tangential counterpart. Contribution of advection and conduction in tangential direction, which is simply known as the convective contribution, or the contribution of conduction in normal direction varies along the interface. Figure 10 shows the distributions of conduction rates at the surface $\left(\dot{q}_{r_{0}}^{\text {cond }}\right)$ and beneath the surface $\left(\dot{q}_{r_{0}-\Delta r}^{\text {cond }}\right)$ along the interface. The difference between these conduction rates is the convective contribution, which is significantly higher when the Marangoni flow is effective. While the contribution of the buoyancy-driven convection to the energy transport is negligible at the interface except the close proximity of the heated substrate, where it reaches its highest value $23 \%$, the contribution of thermocapillary convection is substantial throughout the interface, 59\%-89\% between polar angles $\phi=0-75 \mathrm{deg}$ and $39 \%-73 \%$ between polar angles $\phi=75-90 \mathrm{deg}$.

In experiments, the evaporative heat transfer $\left(\dot{q}^{\text {ev }}\right)$ can be accurately estimated based on the liquid injection rate for a steadily fed droplet. However, liquid or vapor temperature is measured at a finite distance from the interface and this distance cannot be smaller than the radius of measurement probe. Therefore, neither the conduction on liquid side $\left(\dot{q}_{r_{0}}^{\text {cond }}\right)$ nor the conduction on vapor side $\left(\dot{q}^{\text {diff }}\right)$ can be accurately measured at the interface. Accordingly, the conduction measured at a certain distance from the

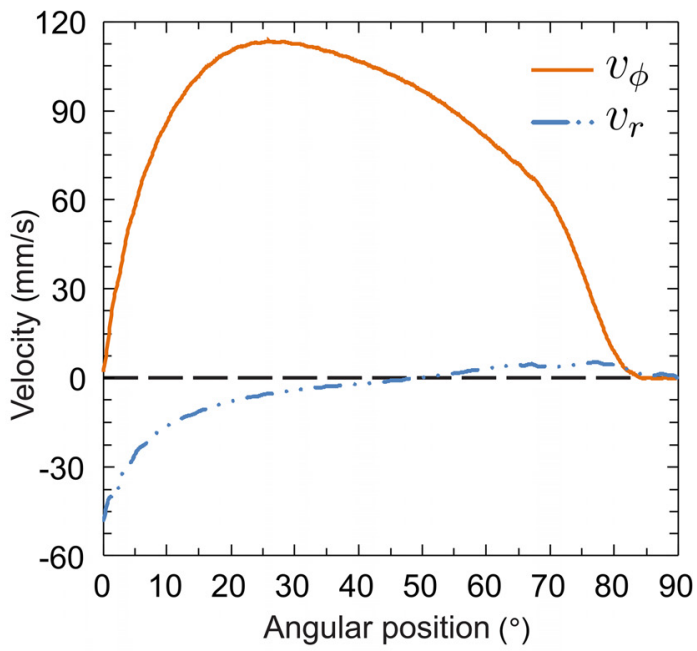

Fig. 9 Distribution of surface velocities for case 3 at $50 \mu \mathrm{m}$ from the interface: (a) w/o thermocapillarity and (b) with thermocapillarity 


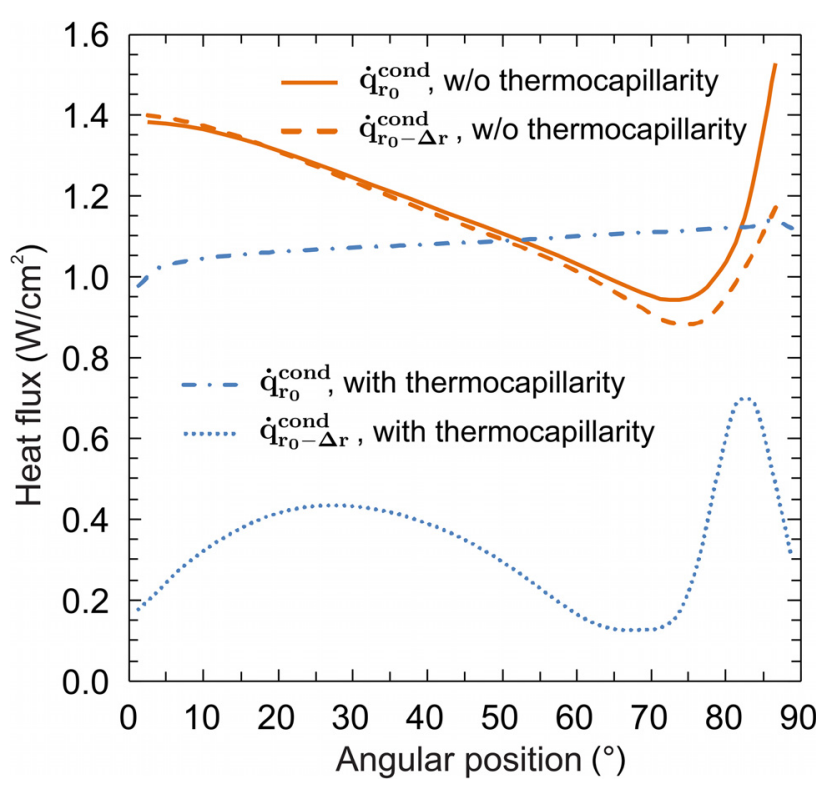

Fig. 10 Distribution of conduction rates for case 3 with and w/o thermocapillarity $(\Delta r=50 \mu \mathrm{m})$

interface $\left(\dot{q}_{r_{0}-\Delta r}^{\text {adv }}\right)$ always deviates from the evaporative heat transfer due to: (i) the tangential convection, especially in the presence of Marangoni flow, (ii) the inaccurately measured conduction on vapor side, and (iii) the omission of radiative heat transfer to the surroundings. Computational modeling presented in this study enables the capturing of the contributions of different heat transport mechanisms at the interface based on an iterative algorithm developed using a widely available commercial software.

\section{Conclusion}

The temperature and flow fields in an evaporating steadily fed spherical water droplet are modeled by solving the full set of Navier-Stokes equations. In order to model the evaporative heat transfer, mass diffusion, and thermocapillarity on the surface, an iterative scheme is developed, which successively solves the flow and temperature fields, updating the evaporative mass flux due to the surface temperature differences until convergence. The simulations reveal a CW flow pattern for the buoyancy driven flow whereas a CCW flow pattern in the presence of thermocapillary convection. In both cases, the magnitude of the evaporating mass is small compared to the circulating mass, which confirms a minor effect of the injected mass on the flow field. The surface temperature distributions obtained with and without the effect of thermocapillarity are compared to experiments. Based on the magnitude of $\mathrm{Ma}$ and the observations from literature, thermocapillary convection is expected to be present in this problem. The simulation results without thermocapillarity, however, are found to capture the distinctive temperature well observed in the experiments.

\section{Acknowledgment}

Authors acknowledge the digital form of the experimental temperature measurement data supplied by Dr. Brendan Macdonald. Y.A. acknowledges the financial support of ASELSAN Inc. under scholarship program for postgraduate studies.

\section{References}

[1] Smalyukh, I. I., Zribi, O. V., Butler, J. C., Lavrentovich, O. D., and Wong, G. C., 2006, "Structure and Dynamics of Liquid Crystalline Pattern Formation in Drying Droplets of DNA," Phys. Rev. Lett., 96(17), p. 177801.
[2] Lim, T., Jeong, J., Chung, J., and Chung, J. T., 2009, "Evaporation of Inkjet Printed Pico-Liter Droplet on Heated Substrates With Different Thermal Conductivity," J. Mech. Sci. Technol., 23(7), pp. 1788-1794.

[3] Sefiane, K., 2010, "On the Formation of Regular Patterns From Drying Droplets and Their Potential Use for Bio-Medical Applications," J. Bionic Eng., 7(S4), pp. S82-S93.

[4] Ebrahimi, A., Dak, P., Salm, E., Dash, S., Garimella, S. V., Bashir, R., and Alam, M. A., 2013, "Nanotextured Superhydrophobic Electrodes Enable Detection of Attomolar-Scale DNA Concentration Within a Droplet by Non-Faradaic Impedance Spectroscopy," Lab Chip, 13(21), pp. 4248-4256.

[5] Kokalj, T., Cho, H., Jenko, M., and Lee, L., 2010, "Biologically Inspired Porous Cooling Membrane Using Arrayed-Droplets Evaporation," Appl. Phys. Lett., 96(16), p. 163703.

[6] Chakraborty, S., Rosen, M. A., and MacDonald, B. D., 2017, "Analysis and Feasibility of an Evaporative Cooling System With Diffusion-Based Sessile Droplet Evaporation for Cooling Microprocessors," Appl. Therm. Eng., 125, pp. 104-110.

[7] Duh, J. C., and Yang, W.-J., 1989, "Numerical Analysis of Natural Convection in Liquid Droplets by Phase Change," Numer. Heat Transfer, 16(2), pp. $129-154$.

[8] Lozinski, D., and Matalon, M., 1993, "Thermocapillary Motion in a Spinning Vaporizing Droplet," Phys. Fluids A Fluid, 5(7), pp. 1596-1601.

[9] Shih, A. T., and Megaridis, C. M., 1996, "Thermocapillary Flow Effects on Convective Droplet Evaporation," Int. J. Heat Mass Tran., 39(2), pp. 247-257.

[10] Ruiz, O. E., and Black, W. Z., 2002, "Evaporation of Water Droplets Placed on a Heated Horizontal Surface," ASME J. Heat Transf., 124(5), pp. 854-863.

[11] Bouchenna, C., Saada, M. A., Chikh, S., and Tadrist, L., 2017, "Generalized Formulation for Evaporation Rate and Flow Pattern Prediction Inside an Evaporating Pinned Sessile Drop,” Int. J. Heat Mass Tran., 109, pp. 482-500.

[12] Girard, F., Antoni, M., Faure, S., and Steinchen, A., 2006, "Evaporation and Marangoni Driven Convection in Small Heated Water Droplets," Langmuir, 22(26), pp. 11085-11091.

[13] Hu, H., and Larson, R. G., 2005, "Analysis of the Effects of Marangoni Stresses on the Microflow in an Evaporating Sessile Droplet," Langmuir, 21(9) pp. 3972-3980.

[14] Ristenpart, W., Kim, P., Domingues, C., Wan, J., and Stone, H., 2007, "Influence of Substrate Conductivity on Circulation Reversal in Evaporating Drops," Phys. Rev. Lett., 99(23), p. 234502.

[15] Xu, X., Luo, J., and Guo, D., 2009 "CCriterion for Reversal of Thermal Marangoni Flow in Drying Drops," Langmuir, 26(3), pp. 1918-1922.

[16] Zhang, K., Ma, L., Xu, X., Luo, J., and Guo, D., 2014, "Temperature Distribution Along the Surface of Evaporating Droplets," Phys. Rev. E, 89(3), p. 032404.

[17] Barash, L. Y., 2015, "Dependence of Fluid Flows in an Evaporating Sessile Droplet on the Characteristics of the Substrate," Int. J. Heat Mass Tran., 84, pp. 419-426.

[18] Hu, H., and Larson, R. G., 2002, "Evaporation of a Sessile Droplet on a Substrate," J. Phys. Chem. B, 106(6), pp. 1334-1344.

[19] Deegan, R. D., Bakajin, O., Dupont, T. F., Huber, G., Nagel, S. R., and Witten, T. A., 1997, "Capillary Flow as the Cause of Ring Stains From Dried Liquid Drops," Nat., 389(6653), pp. 827-829.

[20] Deegan, R. D., Bakajin, O., Dupont, T. F., Huber, G., Nagel, S. R., and Witten, T. A., 2000, "Contact Line Deposits in an Evaporating Drop," Phys. Rev. E, 62(1 Pt B), p. 756

[21] Girard, F., Antoni, M., and Sefiane, K., 2008, "On the Effect of Marangoni Flow on Evaporation Rates of Heated Water Drops," Langmuir, 24(17), pp. 9207-9210.

[22] Kaneda, M., Hyakuta, K., Takao, Y., Ishizuka, H., and Fukai, J., 2008, "Internal Flow in Polymer Solution Droplets Deposited on a Lyophobic Surface During a Receding Process," Langmuir, 24(16), pp. 9102-9109.

[23] Karapetsas, G., Matar, O. K., Valluri, P., and Sefiane, K., 2012, "Convective Rolls and Hydrothermal Waves in Evaporating Sessile Drops," Langmuir, 28(31), pp. 11433-11439.

[24] Strotos, G., Gavaises, M., Theodorakakos, A., and Bergeles, G., 2008 "Numerical Investigation on the Evaporation of Droplets Depositing on Heated Surfaces at Low Weber Numbers," Int. J. Heat Mass Tran., 51(7-8), pp. 1516-1529.

[25] Ait Saada, M., Chikh, S., and Tadrist, L., 2010, "Numerical Investigation of Heat and Mass Transfer of an Evaporating Sessile Drop on a Horizontal Surface," Phys. Fluids, 22(11), p. 112115.

[26] Carle, F., Sobac, B., and Brutin, D., 2013, "Experimental Evidence of the Atmospheric Convective Transport Contribution to Sessile Droplet Evaporation," Appl. Phys. Lett., 102(6), p. 061603.

[27] Yoshitake, Y., Yasumatsu, S., Kaneda, M., Nakaso, K., and Fukai, J., 2009, "Structure of Circulation Flows in Polymer Solution Droplets Receding on Flat Surfaces," Langmuir, 26(6), pp. 3923-3928.

[28] Churchill, S. W., 2002, "Free Convection Around Immersed Bodies," Heat Exchanger Design Handbook, G. F. Hewitt, ed., Begell House, New York.

[29] Akkuş, Y., Cetin, B., and Dursunkaya, Z., 2017, "Modeling of Evaporation From a Sessile Constant Shape Droplet," ASME Paper No. ICNMM2017-5537.

[30] Mahmud, M. A., and MacDonald, B. D., 2017, "Experimental Investigation of Interfacial Energy Transport in an Evaporating Sessile Droplet for Evaporative Cooling Applications," Phys. Rev. E, 95(1), p. 012609.

[31] Robinson, P. J., and Davies, J. A., 1972, "Laboratory Determinations of Water Surface Emissivity," J. Appl. Metrol., 11(8), pp. 1391-1393. 
[32] Mollaret, R., Sefiane, K., Christy, J., and Veyret, D., 2004, "Experimental and Numerical Investigation of the Evaporation Into Air of a Drop on a Heated Surface," Chem. Eng. Res. Des., 82(4), pp. 471-480.

[33] Incropera, F. P., and DeWitt, D. P., 1990, Fundamentals of Heat and Mass Transfer, Wiley, New York.

[34] Vargaftik, N., Volkov, B., and Voljak, L., 1983, "International Tables of the Surface Tension of Water," J. Phys. Chem. Ref. Data, 12(3), pp. 817-820.

[35] Carey, V. P., 1992, Liquid-Vapor Phase Change Phenomena, Hemisphere Publishing House, New York.

[36] Ward, C., and Duan, F., 2004, "Turbulent Transition of Thermocapillary Flow Induced by Water Evaporation," Phys. Rev. E, 69(5 Pt. 2), p. 056308.
[37] Xu, X., and Luo, J., 2007, "Marangoni Flow in an Evaporating Water Droplet," Appl. Phys. Lett., 91(12), p. 124102.

[38] Savino, R., Paterna, D., and Lappa, M., 2003, "Marangoni Flotation of Liquid Droplets," J. Fluid Mech., 479, pp. 307-326.

[39] Duan, F., and Ward, C., 2005, "Surface Excess Properties From Energy Transport Measurements During Water Evaporation," Phys. Rev. E, 72(5), p. 056302.

[40] Duan, F., Badam, V., Durst, F., and Ward, C., 2005, "Thermocapillary Transport of Energy During Water Evaporation," Phys. Rev. E, 72(5), p. 056303.

[41] Ghasemi, H., and Ward, C., 2010, "Energy Transport by Thermocapillary Convection During Sessile-Water-Droplet Evaporation," Phys. Rev. Lett., 105(13), p. 136102. 